Revue Sciences/Lettres

Sciences / Lettres

$4 \mid 2016$

Baba Yaga en chair et en os

\title{
La Baba Yaga sur la route vers l'autre monde : une rencontre cruciale pour le héros du conte
}

Natacha Rimasson-Fertin

\section{(2) OpenEdition \\ 12 Journals}

Édition électronique

URL : http://journals.openedition.org/rsl/913

DOI : $10.4000 /$ rsl. 913

ISSN : 2271-6246

Éditeur

Éditions Rue d'Ulm

Référence électronique

Natacha Rimasson-Fertin, «La Baba Yaga sur la route vers l'autre monde : une rencontre cruciale pour le héros du conte », Revue Sciences/Lettres [En ligne], 4 | 2016, mis en ligne le 16 janvier 2016, consulté le 19 avril 2019. URL : http://journals.openedition.org/rsl/913 ; DOI : 10.4000/rsl.913

Ce document a été généré automatiquement le 19 avril 2019

(c) Revue Sciences/Lettres 


\title{
La Baba Yaga sur la route vers l'autre monde: une rencontre cruciale pour le héros du conte
}

\author{
Natacha Rimasson-Fertin
}

\section{Introduction}

1 Si tout lecteur familier des contes russes a parfaitement présent à l'esprit l'apparence de la fameuse petite isba sur pattes de poule qui sert de domicile à la Baba Yaga, la localisation précise de celle-ci semble moins aisée au premier abord, ne serait-ce que parce qu'elle varie selon les récits.

2 En nous appuyant sur le corpus des contes d'Afanassiev, nous partirons des lieux propres au conte merveilleux ${ }^{1}$ pour y situer plus précisément la demeure de ce personnage aussi emblématique qu'énigmatique qu'est la Baba Yaga. Le but sera de tenter de mieux cerner sa fonction et son identité véritables, à la fois pour le héros voyageur et pour l'auditeurlecteur de ces contes. C'est ainsi qu'Anna-Natalya Malakhovskaya, auteur de l'une des dernières monographies consacrées à la Baba Yaga, s'interroge : « Mais qui la Baba Yaga était-elle vraiment ${ }^{2}[. .$.$] ?»$

Une première étape de notre cheminement s'attachera à resituer la Baba Yaga et sa demeure dans l'univers du conte en accordant une attention particulière aux lieux traversés par le héros. Car même si le genre du conte est caractérisé par son « refus de décrire le trajet ${ }^{3}$ ", son " refus de donner [de l'espace] une description épique ${ }^{4}[.$.$] », on$ parvient tout de même à relever, d'un récit à l'autre, des détails qui, une fois associés, finissent par composer une certaine image de cet univers imaginaire. La question peut donc être posée de la manière suivante : quelle place revient à la Baba Yaga dans la représentation du monde propre au conte? Nous examinerons ensuite cette isba et ce qui s'y passe, avant de nous interroger sur le sens à donner à ces événements, et, plus généralement, sur la portée de ces récits. 


\section{Le lieu de la rencontre avec la Baba Yaga : la frontière avec l'autre monde}

4 Avant d'aborder les textes mettant en scène des héros voyageurs, disons deux mots des récits faisant allusion à l'intrusion de la Baba Yaga dans le monde des hommes lorsqu'elle vient enlever un enfant. À la différence des contes bâtis sur un voyage, les récits comme «Ivachka » (NRS 108-111) ou « Les oies sauvages » (NRS 113) ne situent pas l'habitat de la Baba Yaga dans un autre monde, mais « tout près de l'habitat des hommes : au bord de la rivière, dans la forêt proche où les héros vont simplement chercher des champignons ${ }^{5}$." Sa maison n'a d'ailleurs pas l'allure qu'on lui connaît habituellement et s'apparente davantage à une simple isba.

\subsection{Le voyage : un état d'exception}

5 Les motivations de la quête des héros (ce terme était pris au sens large) voyageurs sont multiples ${ }^{6}$ et mériteraient une étude à part. Les grandes tendances en sont les suivantes : dans le cas des hérö̈nes, il s'agit toujours d'une délivrance et, pour les héros masculins, de la délivrance ou de la quête d'une fiancée, à parts égales.

6 Voyons plutôt quelle est la perception de l'espace propre à ces récits populaires. Dans le conte, l'espace et sa perception s'organisent en fonction des oppositions suivantes: connu/inconnu, familier/étranger. Dans ce contexte, le voyage ${ }^{7}$, dont Propp a montré à quel point il était un élément structurant du conte - « la composition du conte est bâtie sur le déplacement du héros dans l'espace ${ }^{8} »-$ équivaut à une rupture de l'ordre des choses et est d'emblée placé sous le signe de l'extraordinaire' ${ }^{9}$. Un coup d'œil aux termes utilisés dans quelques langues européennes pour désigner le voyage révèle une prédominance de l'idée de rupture, d'effort ${ }^{10}$, de danger aussi, celui-ci étant accentué par la lenteur - et donc la longue durée - des trajets. Dans son étude de la représentation de l'espace au Moyen Âge, Paul Zumthor montre que les durées de déplacement restent à peu près les mêmes au fil des siècles, ce qu'il commente de la manière suivante : «La vitesse relève du merveilleux ; elle ne peut être l'effet que du miracle ou de la magie ${ }^{11}$. " Les contes en donnent des exemples en abondance.

7 En ce qui concerne les traditions russes, l'ethnologue Tatiana Ščepanskaja décrit le statut du voyageur de la façon suivante :

La route est en-dehors de l'ordre divin [...] et, même, s'y oppose... Dans les conceptions traditionnelles, la route est le monde du non-être, où la coutume ne joue pas. Prenant la route, l'individu se retrouve hors de la communauté, il échappe à la zone où pourraient l'atteindre les leviers habituels du tissu social, comme la punition, la pression de l'opinion publique, le prestige, l'encouragement, etc. En route, il est seul, et, donc libre : à la fois en dehors de la communauté et de ses normes ${ }^{12}$.

En d'autres termes, «l'homme voyageant perd tout statut : il n'appartient plus à la société humaine mais à "l'autre monde ${ }^{13}$ " ", et les normes habituelles (vestimentaires, comportementales etc.) n'ont plus cours. De telles représentations, qui peuvent sembler provenir d'un autre temps, ont été recueillies par des anthropologues jusqu'aux années 1980, signe qu'elles sont profondément ancrées dans les mentalités. 
9 Prendre la route, c'est aussi quitter la sphère des ancêtres protecteurs ${ }^{14}$ et s'exposer à toutes sortes de dangers, contre lesquels de nombreuses pratiques avaient pour but de se prémunir, à commencer par le fait de se placer sous la bénédiction parentale, comme le font tous les héros des contes russes ${ }^{15}$. L'ensemble de ces pratiques montre que ce temps du voyage était vécu comme un moment hors du temps quotidien.

10 Cet état d'exception et la situation du voyageur se trouvent synthétisés, au sein du conte, dans le motif de la route, espace ouvert par excellence et lieu de tous les possibles. Dans la dernière version donnée par Afanassiev du conte «Va je ne sais où, rapporte je ne sais quoi » (NRS 212-215), le héros fait allusion lui-même à son statut de voyageur lorsqu'il apostrophe la Baba Yaga qui lui fait part de son intention de le dévorer :

[...] Comment, vieille diablesse! Comment veux-tu manger un voyageur? Un voyageur, c'est osseux et tout noir ; commence par faire chauffer l'étuve, lave-moi

et fais-moi prendre un bain de vapeur, et ensuite, tu me mangeras à ta guise ${ }^{16}$.

11 Avant le fameux repas - qui reste heureusement toujours à l'état de menace -, le bain constitue donc lui aussi un rite de passage, même s'il n'y a pas là, à proprement parler, de réintégration dans la communauté des sédentaires, comme c'était le cas dans la vie quotidienne.

\subsection{La frontière avec l'autre monde}

12 La frontière avec l'autre monde est d'épaisseur variable et diversement matérialisée selon les récits ${ }^{17}$. Elle est le plus souvent doublée d'une clairière, l'ensemble constituant une zone de marge, ou pour citer Nicole Belmont, un «territoire liminaire ${ }^{18}$ ». La Baba Yaga entretient une relation particulière avec la forêt, et sa maison se trouve généralement au cœur de celle-ci, qui l'isole du monde des hommes :

Vassilissa se signa et entra dans la profonde forêt [...]. [Elle] marcha toute la nuit et toute la journée, et c'est seulement le soir qu'elle arriva dans la clairière où se trouvait la maison de la Baba Yaga ${ }^{19}$.

Et comme si l'épaisseur de la forêt ne suffisait pas, la peur semble avoir tracé un cercle autour d'elle: «La Baba Yaga ne laissait personne s'approcher et mangeait les gens comme des poulets ${ }^{20}$.»

13 La forêt se combine parfois avec d'autres obstacles naturels qui contribuent à donner une épaisseur à cette frontière: tantôt il est question d'un profond fossé, tantôt d'une montagne.

Dans « Ilia de Mourom et le dragon » (NRS 310), l'accent est moins mis sur la forêt que sur la localisation de l'isba de la Baba Yaga au sommet d'une montagne, qui prend l'allure d'un vaste plateau. Ainsi, pour se rendre chez le tsar dont la fille est visitée par un dragon, le héros doit gravir une "montagne haute et extrêmement raide et entièrement faite de sable ${ }^{21}$ \%. Une fois là-haut, le héros rencontre non pas une, mais deux Baba Yaga qui lui indiquent comment se rendre chez leur sœur aînée, puis le Brigand-Rossignol, dont le sifflement est si assourdissant qu'il tue tous les voyageurs. Après qu'Ilia a triomphé de ce dernier, la suite de l'action semble se dérouler sur le même plateau, et c'est sans doute là que se trouve aussi le royaume où se rend le héros. C'est du moins une hypothèse, car la descente n'est pas évoquée. Cette vaste zone montagneuse s'apparente donc elle aussi à un monde intermédiaire. Notons que lorsqu'il est dit, plus tôt dans le conte, que le souverain d'Ilia se rend chez le même tsar, le récit passe sous silence les étapes que sont la montagne puis la forêt avec les deux Baba Yaga et le Brigand-Rossignol. 
Voilà pourquoi : la mention de ces obstacles à ce moment-là ne présente pas d'intérêt pour le récit. Ils ne jouent de rôle que comme épreuves pour le héros et non comme lieux en tant que tels : le paysage du conte n'est pas toujours décrit de manière identique et les lieux ne sont évoqués que lorsqu'ils ont une importance pour la progression de l'action.

Par ailleurs, nous avons affaire ici à un triplement du personnage de la Baba Yaga, puisque le héros rencontre successivement trois sœurs, dont l'âge et les pouvoirs vont croissant. La démultiplication de ce personnage, à laquelle s'ajoute le très intimidant Brigand Rossignol, incarnation du mal absolu et de l'ennemi de la terre russe, contribue à dilater la zone de marge, ce qui en fait un véritable no man's land ${ }^{22}$.

Un procédé similaire destiné à figurer l'épaisseur de la frontière se rencontre dans la seconde version de «La plume de Finist, clair faucon» (NRS 235), où le récit s'emploie à accentuer l'effet de l'hyperbole :

Elle marche à travers la sombre forêt, elle marche par-delà des souches et des arbres abattus, tant que ses souliers de fer s'usent, que son bonnet de fer s'élime, que le pain est rongé, mais la belle jeune fille marche encore et encore, et la forêt est de plus en plus noire, de plus en plus dense. Soudain elle voit devant elle une maisonnette de plomb, sur des pattes de poules, qui tourne sans cesse ${ }^{23}$.

Parfois, la maison se situe elle-même «par-delà trois fois neuf pays, dans le trois-foisdixième royaume, de l'autre côté de la rivière de feu ", comme dans " Maria Morevna ${ }^{24}$ " (NRS 159). Comme dans le conte précédent, la rivière de feu est traitée différemment selon les besoins de l'action, et elle joue pleinement son rôle d'ultime frontière entre le monde des hommes et l'autre monde au moment du retour d'Ivan : privée de son foulard magique, la Baba Yaga périt dans les flammes.

\subsection{La maisonnette sur pattes de poule : constantes et variables}

Les nombreuses occurrences de l'isba de la Baba Yaga font de celle-ci un exemple particulièrement éloquent du fonctionnement du conte, au point que V. Propp s'en est servi dans son essai intitulé Les Transformations du conte merveilleux pour illustrer le principe de l'amplification et de la réduction, où « la forme fondamentale est agrandie et complétée par des détails ${ }^{25}$. " Les invariants sont la situation en marge de la forêt et le détail zoomorphe des pattes de poule. Notons que celles-ci sont parfois remplacées ou accompagnées d'autres éléments tout aussi insolites, comme des « cornes de bouc ${ }^{26}$ » dans «Ivan-taurillon».

Dans la seconde version de "La plume de Finist, clair faucon » (NRS 235), l'héroïne se trouve soudain, après avoir longuement cheminé dans la forêt, face à « une maisonnette de plomb, sur des pattes de poules, qui tourne sans cesse ${ }^{27} »$. Propp a montré qu'en réalité la maisonnette ne tourne pas sans cesse, mais seulement lorsqu'on le lui demande - c'est une déformation du motif où le sens initial s'est perdu: "se retourner » est devenu « tourner quand il faut » puis « tourner ${ }^{28}$ ».

20 Dans l'une des sept versions du conte « Le tsar des mers et Vassilissa-la-très-sage » (NRS 224), seule la forêt est évoquée. Cet élément, associé à la formulette prononcée par le héros est ce qui permet d'identifier cette maison comme celle de la Baba Yaga, même en l'absence du détail zoomorphe caractéristique.

Il marche sur la route, la large route, il passe par de vastes prairies, par de riantes plaines et arrive à une sombre forêt. Tout est désert alentour, il n'y a pas âme qui vive. Il n'y a qu'une petite isba, toute seule, l'entrée tournée vers la forêt et le dos vers 
Ivan-fils de marchand. «Maisonnette, maisonnette! lui dit-il, tourne-toi dos à la forêt, et ton entrée face à moi. » La maisonnette lui obéit et se tourna, dos à la forêt, et face à lui. Ivan-fils de marchand entra - la Baba Yaga à la jambe d'os y était étendue d'un coin à l'autre, les tétons pendant de l'autre côté de la plate-bande ${ }^{29}$.

Arrêtons-nous sur un cas particulier. Dans «Les preux Ours, Moustache, Colline et Duchêne » (NRS 141-142), la Baba Yaga et ses filles interviennent comme antagonistes du héros, mais dans un monde souterrain et sans qu'il soit fait mention de la fameuse maisonnette. La désignation du personnage antagoniste comme «Baba Yaga » joue donc sur le complexe d'associations que ce nom éveille dans la mémoire du lecteur, laissant son imagination faire le reste.

\section{L'isba sur pattes de poule : un lieu de mise à l'épreuve du héros}

Voyons à présent ce qui se déroule à l'intérieur de la maison.

\subsection{Un poste-frontière menant dans le monde des morts}

23 Face à cette isba sur pattes de poule, élément familier du lecteur de contes russes mais néanmoins insolite, l'interrogation de Propp est légitime :

Que se passe-t-il donc ici? Pourquoi faut-il faire tourner la petite maisonnette? Pourquoi n'est-il pas possible d'y entrer, tout simplement? Souvent, Ivan a face à lui un mur lisse - «sans fenêtres ni porte » - et l'entrée se trouve du côté opposé. [...] Mais pourquoi ne pas faire le tour de la maisonnette et entrer par l'autre côté ? Manifestement, cela est interdit. De toute évidence, la maisonnette se trouve sur une frontière, visible ou invisible, qu'Ivan ne peut franchir d'aucune manière. On ne peut arriver à cette frontière qu'en passant par la maisonnette, à travers elle, et pour cela, il faut la faire tourner ${ }^{30}[. .$.$] .$

L'examen de différents contes russes et la comparaison avec un mythe amérindien permettent à Propp de conclure que la maison de la Baba Yaga

est tournée, par son côté ouvert, vers le trois-fois-dixième royaume, et, par son côté fermé, vers le royaume accessible à Ivan. Voilà pourquoi Ivan ne peut pas contourner la maisonnette et est obligé de la faire tourner. Cette maisonnette est un poste-frontière ${ }^{31}$.

Un peu plus loin, il complète sa définition de la frontière en rapprochant «l'étrange disposition» de la porte de la maison de la Baba Yaga d'une part avec la coutume scandinave de ne jamais orienter la porte des maisons vers le nord, et, d'autre part avec le fait que, dans l'Edda, la porte de la demeure de la mort (Nastrand ${ }^{32}$ ) est justement tournée vers le nord. Par recoupement, V. Propp conclut que la maison de la Baba Yaga constitue une frontière avec le monde des morts ${ }^{33}$. Il s'agit donc, en quelque sorte, de faire le tri parmi les candidats au passage dans l'autre monde. Cette sélection s'effectue de la manière suivante: la rencontre avec la Baba Yaga constitue en elle-même une épreuve pour le héros.

Si Propp voit dans le motif des pattes de poule un vestige des poteaux zoomorphes qui supportaient la cabane où avait lieu l'initiation ${ }^{34}$, ce détail peut également nous confirmer le lien qui unit le personnage de la Baba Yaga à la mort. Dans son ouvrage sur le paganisme des anciens Slaves, Boris Rybakov évoque en effet une certaine catégorie de morts malfaisants, appelés les nav'i (sing. nav'), qui sont des morts sans baptême, et à qui 
l'on faisait des offrandes. Il précise qu'ils ont «l'aspect d'énormes oiseaux ou de coqs déplumés ${ }^{35}$ » de la taille d'un aigle, qu'ils laissent sur le sol des traces de poule et qu'ils "s'en prennent aux femmes enceintes et aux enfants ${ }^{36}$ ", dont ils sucent le sang. Ces éléments, dont la similitude avec le symbolisme ornithologique de la maison de la Baba Yaga et avec l'hostilité de cette dernière à l'égard des humains est frappante, pourraient fournir une clé pour une meilleure compréhension de ce personnage. La Baba Yaga seraitelle, à l'origine, un de ces mauvais morts ?

Un autre détail nous renseigne sur l'appartenance de la Baba Yaga au monde des morts : la fameuse exclamation par laquelle elle accueille le héros. Comme l'a montré Galina Kabakova, le terme dux dans l'expression russkim duxom paxnet - qui correspond à l'expression française «ça sent la chair fraîche » - désigne l'« odeur, autrement dit ce qui fait reconnaître la nature de l'être et qui est véhiculé par le souffle $e^{37}$ \%. Cette hypersensibilité à l'odeur humaine est une caractéristique commune à tous les êtres de l'autre monde ${ }^{38}$.

\subsection{Des épreuves différentes selon le sexe du héros}

Pour mieux comprendre en quoi consiste cette épreuve, relisons l'échange qui a lieu entre la Baba Yaga et le héros ou l'héroïne. Ivan-taurillon, dans le conte russe du même titre ( Ivan Bykovič », NRS 137), pénètre avec ses compagnons dans la maisonnette «sur pattes de poule, sur cornes de mouton ${ }^{39} »$ :

[...] sur le poêle était allongée la Baba Yaga à la jambe d'os, étendue d'un coin à l'autre, le nez dans le plafond. « Pouah, pouah, pouah! jusqu'au jour d'aujourd'hui je n'avais jamais flairé, jamais vu de mes yeux d'être russe. Mais voici qu'au jour d'aujourd'hui, il en est un pour venir se poser sur ma cuiller, pour rouler tout droit dans ma gueule. - Eh, la vieille, ne te fâche pas, descends donc de ton poêle et assieds-toi sur le banc. Demande-nous où nous allons. Et moi, je te répondrai gentiment ${ }^{40}$.»

La Baba Yaga tient peu ou prou le même discours lorsqu'elle a face à elle une héroïne, comme dans la seconde version de «La plume de Finist, clair faucon » (NRS 234-235). Elle est cependant moins agressive, sans doute parce que la jeune fille s'adresse à elle en l'appelant tendrement " petite grand-mère ${ }^{41}$ » [babusja], ce à quoi elle répond, tout aussi affectueusement : « Eh bien, tu as une longue route à faire, ma petite [maljutka] ».

Ces exemples nous le montrent: les héros et hérö̈nes savent trouver les mots pour intimider et amadouer la Baba Yaga, afin de s'en faire une auxiliaire. On reconnaît là une épreuve comportementale implicite visant à tester le courage dans le cas du héros masculin, et la politesse lorsqu'il s'agit d'une héroïne.

31 Cette épreuve revêt diverses formes et peut être plus ou moins intentionnelle selon les textes. Elle aboutit toujours à un changement de fonction de la Baba Yaga qui, de gardienne et d'adversaire, se fait guide et auxiliaire du héros.

Dans certains récits, la Baba Yaga, bien moins agressive qu'à son habitude, endosse d'emblée un rôle de guide, et n'est pas dépourvue de tendresse. Dans la première version du conte « Le tsar des mers et Vassilissa la très-sage » (NRS 219-226), voici ce qu'elle dit au héros une fois que celui-ci lui a appris l'objet de sa quête :

Va, mon enfant [ditjatko], au bord de la mer. Douze cigognes voleront jusque-là ; elles se changeront en belles filles et se baigneront. Approche-toi tout doucement et prends à l'aînée sa chemise ${ }^{42}$. 
Cette fonction d'auxiliaire et de guide se rencontre notamment dans les contes où la vieille femme est la maîtresse des animaux ou des vents. Ainsi, dans "La princesse ensorcelée » (NRS 271-272, version $n^{\circ} 272$ ), le héros, un soldat, part en tapis volant à la recherche du royaume où se trouve sa fiancée magique, et se rend successivement chez trois Baba Yaga, trois sœurs, dont la dernière habite « au bout du monde » :

Il y avait là une maisonnette, et au-delà, il n'y avait que de l'obscurité [t'ma kromešnaja], on ne voyait rien! « Eh bien! se dit-il, si je n'obtiens pas de réponse ici, ensuite, je peux plus aller nulle part ! [bolše letet' nekuda $\left.a^{43}\right]$ »

Au bout du monde, il n'y a donc, en somme, que l'obscurité, le néant. Cette dernière Baba Yaga est la maîtresse des vents et offre son aide au soldat. C'est le vent du sud, le dernier arrivé, qui apprend au soldat où trouver son épouse en racontant qu'il revient d'un nouveau royaume. Voici sa description du temps nécessaire pour s'y rendre :

À pied, on mettrait trente ans, un oiseau mettrait dix ans, mais si je me mets à souffler, nous y serons en trois heures ${ }^{44}$.

\subsection{Une rencontre à valeur initiatique}

Parfois, le séjour chez la Baba Yaga se prolonge et l'épreuve comportementale est remplacée par une autre, qui a pour enjeu la vie du héros : dans « Maria Morevna » (NRS 159), le héros se rend chez la Baba Yaga pour obtenir un cheval magique capable de battre à la course celui de Kochtcheï-l'Immortel, afin de délivrer son épouse surnaturelle.

«Je viens mériter chez toi un cheval de preux. - Très bien, tsarévitch! Mais chez moi, le service ne dure pas un an, mais seulement trois jours; si tu réussis à garder mes cavales, je te donnerai un cheval de preux, mais si tu n'y arrives pas, ne te fâche pas, mais ta tête ira coiffer le dernier poteau. » Le tsarévitch Ivan acquiesça ; la Baba Yaga lui donna à manger et à boire, puis elle lui ordonna de se mettre au travail ${ }^{45}$.

Le héros viendra à bout de cette épreuve grâce à des animaux reconnaissants qu'il a épargnés auparavant.

Un autre exemple d'épreuve particulièrement intéressant se rencontre dans le célèbre conte «Vassilissa-la-très-belle » (NRS 104), qui est aussi celui où la maison de la Baba Yaga est décrite avec le plus de précision, avec notamment le détail particulièrement morbide des poteaux coiffés de crânes humains, que l'on distingue nettement dans l'illustration de ce conte par Ivan Bilibine (1899). Le peintre a également fait le choix de représenter l'isba juchée sur des pattes de poule, alors que ce détail n'est pas mentionné dans la description citée plus haut, parce qu'il est indissociable du personnage dans l'imaginaire collectif. 
Figure 1 - Ivan Bilibine, Vassilissa la très Belle sortant de la maison de Baba Yaga. Illustration pour le conte « Vassilissa la très Belle », 1899.

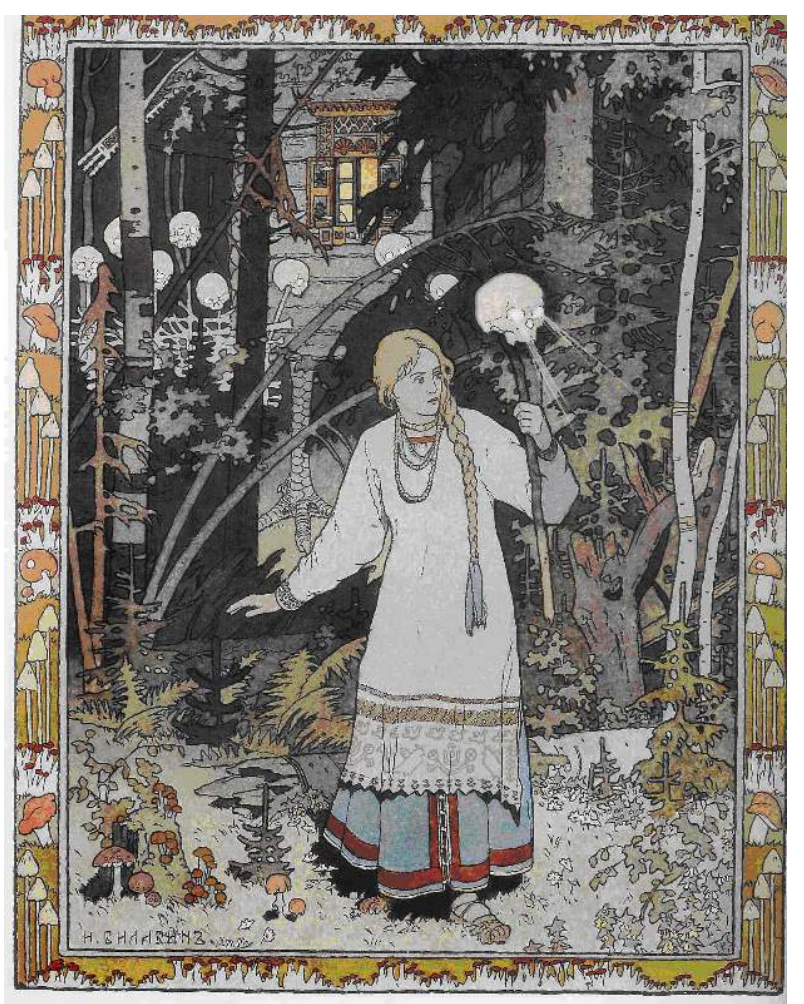

O. Semënov, Ivan Bilibin. Rasskaz o xudožnike-skazočnike [Ivan Bilibine. Histoire d'un peintre-conteur], Moscou, éditions Detskaja Literatura, 1996, p. 23.

Après avoir volontairement laissé s'éteindre le feu de la maison, la marâtre envoie l'héroïne chercher du feu chez la Baba Yaga, dans l'espoir de se débarrasser d'elle. Au lieu d'être dévorée, l'héroïne rentre saine et sauve, mais en plus, ce voyage dans l'autre monde remplit une fonction réparatrice, puisqu'il rétablit la justice en infligeant à la marâtre le châtiment qu'elle mérite : le feu rapporté par Vassilissa réduit cette dernière et ses filles en cendres.

Notons que dans ce récit, l'héroïne ne pénètre pas dans l'isba de la Baba Yaga en la faisant tourner. Après avoir traversé la forêt pendant un jour et demi, et vu passer les trois cavaliers, elle attend la maîtresse des lieux près du portail.

En plus de devoir surmonter sa peur, la jeune fille est soumise à des épreuves bien plus complexes. La première consiste à s'acquitter des tâches domestiques imposées par la Baba Yaga, qui s'apparentent par leur ampleur à des tâches impossibles, mais dont Vassilissa vient à bout avec l'aide de sa poupée magique et de la bénédiction maternelle, la première étant en quelque sorte l'incarnation de la seconde.

La seconde épreuve a lieu pendant l'échange qui clôt son séjour chez la Baba Yaga. Celleci encourage la jeune fille à lui poser des questions, tout en la mettant en garde contre la question de trop, car «toute question n'est pas bonne à poser ${ }^{46}$ ». Cette mise à l'épreuve est implicite et consiste à ne pas violer le tabou ${ }^{47}$ - tacite - en interrogeant la vieille sur ce qui se passe à l'intérieur de la maison.

42 Cette fois, Vassilissa sort victorieuse de l'épreuve parce qu'elle sait se contenter d'observer : elle se garde bien d'interroger la Baba Yaga sur les trois mystérieuses paires 
de mains qu'elle a vues à l'intérieur de l'isba, ce pourquoi celle-ci la félicite. Mais surtout, comme le souligne A.-N. Malakhovskaya, parce qu'elle s'en sort sans l'aide de sa poupée, qu'elle ne peut consulter au moment où elle parle avec la Baba Yaga ${ }^{48}$.

En ayant vu ce qu'elle a vu et en ayant triomphé de sa peur, Vassilissa a été initiée aux tâches typiquement féminines propres à cette société patriarcale, et relevant de la troisième fonction dumézilienne de fertilité/fécondité : non seulement à celles relevant du ménage et de la préparation des repas, mais aussi et surtout à celles qui la mèneront vers l'accomplissement du destin annoncé par son nom (« Vassilissa » est dérivé du grec basileus, le roi), grâce au filage et au tissage. On se souvient que dans le second épisode du conte, Vassilissa est recueillie par une vieille femme chez qui elle tisse des chemises; la finesse de celles-ci lui vaudra d'être appelée chez le tsar qui l'épousera.

Le fait que le héros ou l'héroïne parviennent à s'acquitter de l'épreuve qui leur est imposée contribue à les identifier comme tels. Il s'agit donc là d'épreuves "qualifiantes ", pour reprendre les termes de Nicole Belmont ${ }^{49}$.

Les contes russes comportent également d'autres personnages féminins qui ne sont désignés que comme des "vieilles femmes", mais que plusieurs éléments permettent d'assimiler à la Baba Yaga et à sa fonction d'initiatrice : leur grand âge, le fait qu'elles ont un habitat similaire (une maisonnette à la lisière ou au cœur de la forêt, avec parfois la même description), et, surtout, leur manière de s'adresser au héros. On rencontre même, dans «Ivan-de-la-chienne et le Sylvain Blanc» (NRS 139), un équivalent masculin de la Baba Yaga : « un vieillard âgé dans un mortier, qui se pousse avec un pilon ${ }^{50}$ ». Cependant, son comportement évoque davantage celui du petit homme grand d'un pouce, évoqué plus haut. Cet exemple illustre parfaitement le caractère interchangeable et polymorphe de ces êtres surnaturels, qui remplissent la même fonction, celle de figures du destin, de passeur entre les mondes et d'initiateur.

L'analyse de tous ces exemples permet donc de pointer une nouvelle fois l'ambivalence profonde du personnage de la Baba Yaga, à laquelle les chercheurs ont tenté d'apporter différentes explications, bien souvent sans parvenir à surmonter réellement les contradictions qui la caractérisent. L'interprétation la plus pertinente semble être celle d'Anna-Natalya Malakhovskaya, qui voit dans la Baba Yaga non plus simplement la déesse-mère archaïque caractérisée par la dichotomie bien $/ \mathrm{mal}^{51}$, mais plutôt une divinité dont les différents rôles correspondent aux trois fonctions duméziliennes, qui sont respectivement la sagesse et le lien au sacré (et aux divinités magiques), la fonction guerrière, et la fonction de fertilité/fécondité qui est aussi liée à la mort ${ }^{52}$.

\section{Des récits porteurs d'un « sens latent ${ }^{53}$ »}

Quel sens faut-il donc donner à ces récits relatant un voyage dans l'autre monde, c'est-àdire dans l'au-delà, le monde des morts ? Par-delà leur fonction compensatrice et celle de divertissement, propres au genre du conte, ces récits-là seraient-ils eux-mêmes porteurs d'une fonction initiatique et étiologique?

On se souvient que Vassilissa-la-très-belle, dans le conte éponyme (NRS 104), est chassée par la Baba Yaga une fois qu'elle lui a révélé la nature de son auxiliaire magique :

«[...] À présent, c'est moi qui vais te poser une question : comment fais-tu pour réussir à faire le travail que je te donne? - C'est la bénédiction de ma mère qui m'aide, 
répondit Vassilissa. - Voilà donc ce qu'il en est ! Puisqu'il en est ainsi, va-t-en d'ici,

fille bénie que tu es ! Je n'ai pas besoin de ceux qui sont bénis ${ }^{54}$ !»

$49 \mathrm{Au}$ delà de sa fonction compensatrice, ce récit est donc porteur d'une dimension didactique. Cette seconde fonction transparaît dans les vertus incarnées par le personnage de Vassilissa, qui réunit à la fois piété - au sens traditionnel de ce terme - et piété filiale (à travers sa fidélité à la dernière recommandation de sa mère), mais aussi la docilité, la serviabilité, ainsi que le courage ou plutôt la confiance, forte qu'elle est de la bénédiction maternelle. Car contre celle-ci, la Baba Yaga, toute puissante qu'elle est, ne peut rien ${ }^{55}$.

50 Ce dernier motif, dont le conte de Vassilissa donne une illustration particulièrement prégnante, met en évidence à quel point le personnage de la Baba Yaga renvoie à des représentations archaïques et préchrétiennes, puisque c'est finalement le lien aux ancêtres protecteurs, par le biais de la bénédiction maternelle, qui assure le salut de l'héroïne. Ainsi, ce conte illustre-t-il à merveille la notion de «double foi » utilisée pour caractériser le christianisme populaire propre à la Russie ${ }^{56}$.

51 Revenons enfin, pour clore notre réflexion, aux contes évoqués à l'orée de cet article, qui avaient pour motif central l'enlèvement d'enfants, afin de tenter d'élucider leur fonction. Comment expliquer, en effet, cette intrusion de la Baba Yaga dans le monde des hommes, qu'elle soit directe, ou qu'elle se fasse par le biais d'intermédiaires comme les oies sauvages? Si nous avons vu, à propos du conte «Les oies sauvages » (NRS 113), que loin d'être en danger chez la Baba Yaga ${ }^{57}$, le petit frère y jouait avec des pommes d'or, c'est davantage son enlèvement qui fait l'objet d'une crainte que son sort. La peur porte donc bien plus sur le trouble de l'ordre des choses que représente ce rapt - d'autant que le conte met également l'accent sur la peur de la fillette, qui craint d'être punie par ses parents pour son défaut de surveillance.

52 Ce récit serait donc porteur, comme d'autres, d'une fonction étiologique, l'intrusion du personnage inquiétant de la Baba Yaga ou de ses émissaires ailés, les oies sauvages, étant destinée à apporter une réponse à des phénomènes que l'entendement se refusait à expliquer, tels que la mortalité infantile ${ }^{58}$ - on se souvient qu'il est dit, au début du conte «Les oies sauvages », que ces-dernières « enlevaient les petits enfants ${ }^{59}$ ".

\section{Conclusion}

La Baba Yaga nous est donc apparue à la fois comme gardienne de la frontière avec l'autre monde, mais aussi comme passeuse vers celui-ci et comme guide, comme être à la frontière des mondes, mi-vivant, mi-mort, mais aussi des règnes, mi-animal, mi-humain.

Comme l'a noté N. Novikov, «dans la figure de la Baba Yaga, sans doute comme dans aucun autre personnage de contes, se sont déposées, d'une manière bizarre et particulière, des strates culturelles séculaires, et les démêler n'est pas une tâche facile ${ }^{60}$ ...». On peut donc voir en elle un personnage-palimpseste, une divinité alliant les dimensions à la fois chthonienne et spirituelle, et présidant à la destinée des hommes.

Quant à la représentation de l'univers, la Weltanschauung qui se dégage des contes, au sein de laquelle la frontière, si étroitement liée à la Baba Yaga, joue une place centrale, on peut parler d'une géographie à trois pôles qui sont ce monde-ci, l'autre monde et l'entredeux-mondes ; entre ces lieux, le passage est possible dans un sens comme dans l'autre. 
Ce passage, géographique en apparence, si l'on considère le voyage accompli par les héros et héroïnes, est en réalité un passage à une " altérité ontologique ${ }^{61}$ ", conformément à un procédé cher au genre du conte, celui de l'extériorisation ${ }^{62}$. En effet, sous l'apparence de simples histoires visant le divertissement, ces récits narrent de manière imagée les changements qui s'opèrent lors du passage à l'âge adulte, tant à l'échelle individuelle que du point de vue du statut social, et sont donc eux-mêmes investis d'une fonction initiatrice.

\section{BIBLIOGRAPHIE}

Afanassiev, Alexandre Nikolaiévitch, Narodnyje Russkije Skazki, (3 t.), édition établie, préfacée et annotée par V. Propp, Moscou, Gosudarstvennoe Izdatel'stvo Xudožestvennoj Literatury, 1957.

Becker, Richarda, Die weibliche Initiation im ostslawischen Zaubermärchen, Wiesbaden, Otto Harrassowitz, 1990.

Belmont, Nicole, «Les seuils de l'autre monde dans les contes populaires français », Cahiers de littérature orale, $\mathrm{n}^{\circ}$ 39-40, 1997, p. 61-79.

-, Poétique du conte. Essai sur le conte de tradition orale, Paris, Gallimard, 1999.

-, «Manipulation et falsification des contes traditionnels par les cultures lettrées », ethnographiques.org, numéro 26, 2013, Sur les chemins du conte : http:// www.ethnographiques.org/2013/Belmont

Čistov, Kirill, « Baba-Jaga », Märchenspiegel, nº 7, 1996, p. 38-42.

Conte, Francis, L'Héritage païen de la Russie, Paris, Albin Michel, 1997.

Dumézil, Georges, Mythe et épopée, Paris, Gallimard, 1968.

—, Les Dieux souverains des indo-européens, Paris, Gallimard, 1977.

Hetmann, Frederik, « Die keltische Anderswelt als Reich der Wunscherfüllung und Phantasie », in Lox, Harlinda, Volkmann, Helga et Bücksteeg, Thomas (éd.), Homo faber - Handwerkskünste in Märchen und Sagen/Verlorene Paradiese - Gewonnene Königreiche. Forschungsbeiträge aus der Welt der Märchen, Krummwisch, Königsfurt, 2005, p. 164-193.

Horn, Katalin, « Der Weg », in J. Janning et alii (éd.), Die Welt im Märchen, Kassel, Erich Röth Verlag, 1984, p. 22-37.

Kabakova, Galina, « Les représentations des odeurs dans la culture populaire slave », Cahiers slaves, $\mathrm{n}^{\circ} 1$ 1, 1997, p. 205-216.

Lecouteux, Claude, La Maison et ses génies, Paris, Imago, 2000.

—, Dictionnaire de mythologie germanique, Paris, Imago, 2005.

Malakhovskaya, Anna-Natalya, Nasledie baby-yagi. Religioznye predstavleniya, otražennye v volšebnoj skazke, i ix sledy v russkoj literature XIX-XX vv., Saint-Pétersbourg, Aleteia, 2006. (en russe) 
Novikov, Nikolaï, Obrazy vostočnoslavjanskoj volšebnoj skazki [Les figures du conte merveilleux des Slaves orientaux], Moscou, Nauka, 1974. (en russe)

Propp, Vladimir, Morphologie du conte, suivi de Les transformations du conte merveilleux, et de E. Mélétinski, L'Étude structurale et typologique du conte, traductions de Marguerite Derrida, Tzvetan Todorov et Claude Kahn, Paris, Le Seuil, coll. « Poétique », 1970.

-, Les Racines historiques du conte merveilleux, trad. fr. Lise Gruel-Apert, Paris, Gallimard, coll. « NRF », 1983.

Rimasson-Fertin, Natacha, L'Autre monde et ses figures dans les Kinder - und Hausmärchen des frères Grimm et les Contes populaires russes d'A. N. Afanassiev, université Stendhal-Grenoble 3, thèse de doctorat, 2008.

- , « Putešestvie v inoe carstvo kak osnova struktury skazki : sravnitel'nyj analiz skazok brat'ev Grimm i A. N. Afanas'eva » [Le voyage dans l'autre monde comme élément structurant du conte : étude comparée des contes des frères Grimm et d'A. N. Afanassiev] in Tradicionnaya kultura [La culture traditionnelle, almanach scientifique], $\mathrm{n}^{\circ}$ 58, 2015 (2), p. 104-119. (voir : http:// www.folkcentr.ru/izdaniya/zhurnal-tradicionnaya-kultura/) (en russe)

Rybakov, Boris, Le Paganisme des anciens Slaves, Paris, PUF, 1994 (Moscou 1981), coll. « Ethnologies », trad. fr. Lise Gruel-Apert.

Ščepanskaja, Tatiana, « La culture de la route dans la Russie du Nord », Ethnologie française, XXVI, 4, 1996, p. 677-690.

Zumthor, Paul, La Mesure du monde. Représentation de l'espace au Moyen Âge, Paris, Seuil, coll. « Poétique », 1993.

\section{NOTES}

1. Cet article s'inscrit dans la continuité de nos précédents travaux sur l'autre monde dans les recueils de contes d'Afanassiev et des frères Grimm. Voir N. Rimasson-Fertin, L'Autre monde et ses figures dans les Kinder - und Hausmärchen des frères Grimm et les Contes populaires russes d'A. $N$. Afanassiev.

2. « А КЕМ ЖЕ БЫЛА БАБА-ЯГА НА САМОМ ДЕЛЕ [...]?», in A.-N. Malakhovskaya, Nasledie baby-yagi. Religioznye predstavleniya, otražennye $v$ volšebnoj skazke, i ix sledy v russkoj literature XIX-XX vv., p. 5.

3. V. Propp, Les Racines historiques du conte merveilleux, p. 57.

4. Ibid.

5. N. Novikov, Obrazy vostočnoslavjanskoj volšebnoj skazki, p. 74.

6. N. Rimasson-Fertin, L'Autre monde et ses figures..., p. 390-396.

7. Sur le voyage chez Afanassiev et Grimm, voir N. Rimasson-Fertin, «Putešestvie v inoe carstvo kak osnova struktury skazki », p. 104-119.

8. V. Propp, Les Racines historiques..., op. cit., p. 56.

9. Voir également K. Horn, « Der Weg », p. 23.

10. P. Zumthor, La Mesure du monde. Représentation de l'espace au Moyen Âge, p. 167.

11. Ibid., p. 172.

12. T. Ščepanskaja, « La culture de la route dans la Russie du Nord », p. 678.

13. Ibid., p. 677.

14. Voir C. Lecouteux, La Maison et ses génies.

15. Cette coutume existe aussi dans le monde germanophone, mais elle ne transparait pas dans les contes. 
16. A. N. Afanassiev, Narodnye russkie skazki (cité NRS infra), p. 156-157. En l'absence d'indication contraire, tous les soulignements sont le fait de l'auteur de l'article.

17. Voir N. Rimasson-Fertin, L'Autre monde et ses figures..., op. cit., p. 75-126.

18. N. Belmont, « Les seuils de l'autre monde dans les contes populaires français », p. 64.

19. NRS, t. 1, p. 161.

20. NRS, t. 1, p. 160.

21. NRS, t. 3, p. 11.

22. Le même effet de dilatation de la frontière est parfois obtenu grâce à un doublement ou à un triplement du personnage du gardien. Le « Conte du jeune preux, des pommes de jouvence et de l'Eau de la Vie » (NRS 171-178) en offre plusieurs exemples. Dans la version $n^{\circ} 176$, Elena-la-trèsbelle, qui s'élance à la poursuite du héros qui a pénétré chez elle, reproche à ses sœurs de l'avoir laissé passer, par une phrase qui rend manifeste leur fonction de gardiennes : «Pourquoi donc vous a-t-on placées ici ? » Dans la version $n^{\circ} 178$, trois Baba Yaga jouent le même rôle.

23. NRS, t. 2, p. 243.

24. NRS, t. 1, p. 379.

25. V. Propp, Morphologie du conte, p. 185.

26. NRS, t. 1, p. 279.

27. NRS, t. 2, p. 243.

28. V. Propp, Morphologie du conte, op. cit., p. 186.

29. NRS, t. 2, p. 199-200.

30. V. Propp, Les Racines historiques du conte merveilleux, op. cit., p. 72.

31. Ibid., p. 73.

32. Ce nom, qui signifie «Rivage des cadavres », désigne une des demeures de Hel, la déesse des enfers. Voir C. Lecouteux, Dictionnaire de mythologie germanique, p. 119-120 et p. 173.

33. Voir V. Propp, Les Racines historiques..., op. cit., p. 154.

34. Ibid., p. 158-159.

35. B. Rybakov, Le Paganisme des anciens Slaves, p. 35.

36. Ibid.

37. G. Kabakova, «Les représentations des odeurs dans la culture populaire slave», p. 206. L'auteur rappelle notamment les différents sens du terme dux: «Dux est, selon les contextes, souffle, souffle de vent, air (vozdux), alcool, vapeur, respiration, inspiration, esprit en tant que substance incorporelle opposée à la chair, être immatériel, démon, souffle créateur de Dieu, essence, force principale, univers spirituel, confession. »

38. Ibid., p. 212-213.

39. NRS, t. 1, p. 279.

40. V. Propp, Les Racines historiques..., op. cit., p. 78.

41. NRS, t. 2, p. 244. Comme le note très justement R. Becker, le terme baba a, à lui seul, la signification de "grand-mère " dans toutes les langues slaves, même s'il a tendance, depuis toujours, à élargir son sens pour désigner les femmes mariées. Voir R. Becker, Die weibliche Initiation im ostslawischen Zaubermärchen, p. 114.

42. NRS, t. 2, p. 174.

43. NRS, t. 2, p. 351.

44. NRS, t. 2, p. 352.

45. NRS, t. 1, p. 380-381.

46. NRS, t. 1, p. 163.

47. A.-N. Malakhovskaya, Nasledie Baby Yagi, op. cit., p. 32.

48. Ibid., p. 34 .

49. N. Belmont, Poétique du conte. Essai sur le conte de tradition orale, p. 185.

50. NRS, t. 1, p. 293.

51. Cet aspect a notamment été souligné par R. Becker, voir R. Becker, op. cit., p. 162. 
52. A.-N. Malakhovskaya, op. cit., p. 10-13 et G. Dumézil, Mythe et épopée 1, et, du même, Les Dieux souverains des indo-européens.

53. N. Belmont, « Manipulation et falsification des contes traditionnels par les cultures lettrées ». 54. NRS, t. 1, p. 163.

55. Dans son ouvrage, A.-N. Malakhovskaya propose une lecture très intéressante de la questionclé de la Baba Yaga. Celle-ci serait, semble-t-il, en quête d'une héritière digne de prendre sa succession, comme le laissent entendre les mots avec lesquels elle chasse Vassilissa une fois qu'elle sait que sa force réside en la bénédiction de sa mère : " Je n'ai pas besoin de ceux qui sont bénis » - ce qui sous-entend bien qu'elle a besoin de quelqu'un, dans un but qui n'est pas dévoilé. Pour l'auteur, le prénom même de Vassilissa la place au même rang que toutes les héroïnes qui se trouvent être des filles ou des nièces de la Baba Yaga. Voir A.-N. Malakhovskaya, op. cit., p. 34-35. Ceci vient renforcer le choix de traduction fait par L. Gruel-Apert pour le nom de cette héroïne, qu'elle appelle « Vassilissa la Magique ».

56. F. Conte, L'Héritage païen de la Russie, p. 23-24.

57. Nous tenons à remercier ici Evelyne Cevin pour son interprétation très éclairante de ce récit.

58. Voir F. Hetmann, « Die keltische Anderswelt als Reich der Wunscherfüllung und Phantasie », p. 174.

59. NRS, t. 1, p. 185.

60. N. Novikov, op. cit., p. 180. Voir aussi, K. Čistov, « Baba-Jaga », p. 38-42.

61. Concernant cette différence ontologique entre les deux mondes, voir N. Belmont, « Les seuils de l'autre monde dans les contes populaires français ", op. cit., et N. Rimasson-Fertin, L'Autre monde et ses figures..., op. cit., p. 460.

62. N. Rimasson-Fertin, L'Autre monde et ses figures..., op. cit., p. 460-461.

\section{RÉSUMÉS}

Cet article s'appuie sur une étude du recueil d'Afanassiev dans sa langue d'origine et vise à situer précisément la demeure de la Baba Yaga dans l'espace du conte pour définir son identité véritable et sa fonction. La rencontre avec cet être a lieu à la frontière avec l'autre monde. La Baba Yaga apparaît, quant à elle, comme un personnage ambivalent, gardienne de la frontière, mais aussi donatrice et initiatrice du héros. Quel sens donner à ces récits relatant un voyage dans l'autre monde ? Loin d'être un simple divertissement, ils sont eux-mêmes investis d'une fonction initiatique et étiologique.

This article, based on a study of Afanassiev's tales collection, aims at locating accurately Baba Yaga's hut in the space of the fairy tale, in order to define her real identity and her function. The hero always meets her at the border of the Otherworld, and she appears as an ambivalent character, which is a guardian of the border, as well as a donator and an initiator of the hero. What is the meaning of these tales about a journey to the Otherworld? Far from being a simple entertainment, they also have an initiating and an etiological dimension. 
INDEX

Mots-clés : Baba Yaga, autre monde, géographie mythique, Weltanschauung, voyage, frontière, initiation, au-delà, épreuve, destin, mort

Keywords : otherworld, mythical geography, travel, boundary, world beyond, destiny, death

\section{AUTEUR}

\section{NATACHA RIMASSON-FERTIN}

Maître de conférences en langue et littérature allemandes à l'Université Grenoble-Alpes et chercheur à l'ILCEA4 (Institut des langues et des cultures d'Europe, Amérique, Afrique, Asie et Australie) depuis 2009, Natacha Rimasson-Fertin est une ancienne élève de l'École normale supérieure de Fontenay-Saint-Cloud. D'origine russe et agrégée d'allemand, elle est titulaire d'un doctorat d'études germaniques, consacré au motif de l'autre monde dans les contes de Grimm et d'Afanassiev (2008). Auteur de la nouvelle traduction et édition critique des Contes pour les enfants et la maison des frères Grimm (José Corti, 2009), qui a obtenu le prix Halpérine-Kaminsky Découverte, de la Société des gens de lettres, et un prix de l'Académie française en 2010, ses recherches portent sur les contes populaires allemands et russes : croyances et traditions populaires, folklore et identité nationale, contes et imaginaire, adaptations littéraires, iconographiques et cinématographiques. 